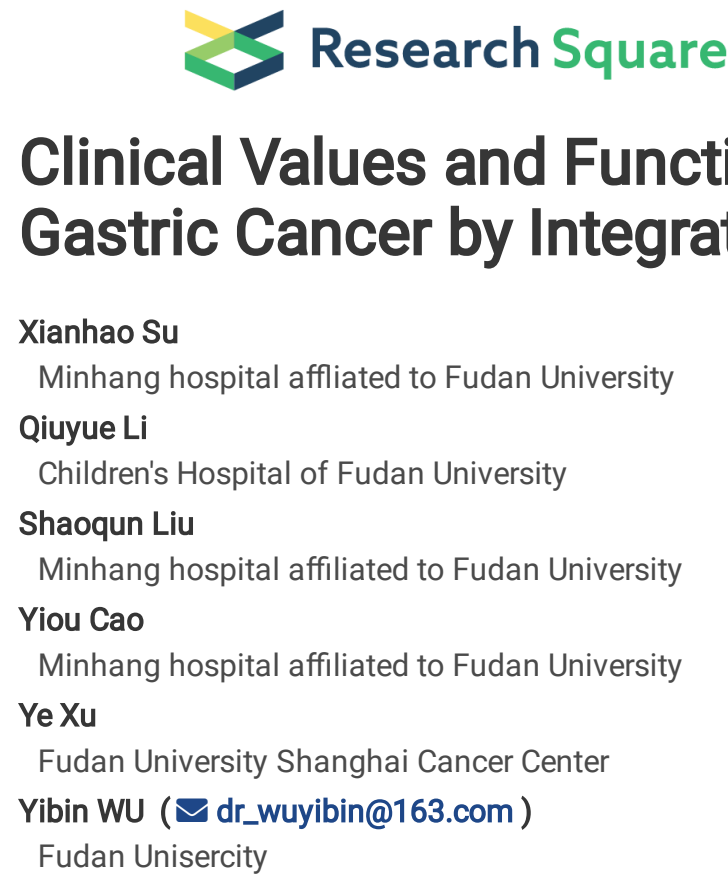

\author{
Xianhao Su \\ Minhang hospital affliated to Fudan University \\ Qiuyue Li \\ Children's Hospital of Fudan University \\ Shaoqun Liu \\ Minhang hospital affiliated to Fudan University \\ Yiou Cao \\ Minhang hospital affiliated to Fudan University \\ Ye Xu \\ Fudan University Shanghai Cancer Center \\ Yibin WU ( $\nabla$ dr_wuyibin@163.com ) \\ Fudan Unisercity
}

\title{
Clinical Values and Functional Analysis Of KMT2A/B/C/D/E/F in Gastric Cancer by Integrated Bioinformatics Analysis
}

\section{Research article}

Keywords: KMT2s, Gastric cancer, Bioinformatics, Clinical value, Function

Posted Date: April 3rd, 2021

DOI: https://doi.org/10.21203/rs.3.rs-378432/v1

License: (c) (i) This work is licensed under a Creative Commons Attribution 4.0 International License. Read Full License 


\section{Abstract}

$\mathrm{KMT} 2 \mathrm{~A} / \mathrm{B} / \mathrm{C} / \mathrm{D} / \mathrm{E} / \mathrm{F}$ have been identified be involved in the tumor progression of gastric cancer (GC). However, the clinical significance and function of the 6 KMT2s have not yet been analyzed. Here, we investigated the transcriptional and survival data of KMT2s family genes in GC based on integrated bioinformatics analysis. CBioPortal was used to analyze the alteration frequency and functional networks of KMT2s in GC. And GO and KEGG pathways were also identified. Gene enrichment analysis was performed to investigate the networks of kinase, miRNAs and transcription factor target. We found that KMT2s were highly expressed in GC, and associated with tumor stages and grades. Besides, higher KMT2s expression were significantly associated with shorter overall survival (OS), progression-free survival (FP) and postprogression survival (PPS). Also, promoter methylation levels of KMT2s were positively associated with KMT2s expression and clinical data. Moreover, high mutation rate of KMT2s (40\%) was also observed in GC patients. Functional networks analysis suggested that KMT2s might regulate histone lysine methylation and chromatin organization via PI3K/AKT and MAPK signaling pathways involving in some kinase, miRNA and transcription factor. Taken together, these findings suggested that KMT2A/B/C/D/E/F may be prognostic and therapeutic biomarkers of patients with GC.

\section{Introduction}

Gastric cancer (GC) is the fifth most common cancer and the third leading cause of cancer-related death worldwide[1, 2]. In advanced GC patients, the purpose of chemotherapy is to shrink tumor size, reduce tumor growth, and inhibit tumor metastasis. The majority of patients with advanced GC are initially responsive to the combined chemotherapy. However, the patients eventually experience tumor recurrence or metastasis partially due to drug resistance, and the 5 year survival rate is only $30 \%$ in advanced GC patients[3]. Fortunately, some of those patients are responsive to cancer-targeted therapy. Thus, identifying novel cancer-related genes is essential to optimizing current therapeutic strategies.

The lysine methyltransferase 2 (KMT2, also known as mixed lineage leukemia (MLL)) family methylates histone H3 lysine 4 (H3K4) to promote genome accessibility and transcription $[4,5]$. The KMT2 family of histone modifying proteins play essential roles in regulating developmental pathways, and mutations in the genes encoding these proteins have been identified being involving in various human malignancies[6], including breast cancer[7], bladder cancer[8], lung cancer[9], blood cancer[10], large intestinal cancer[11] and kidney cancer[12].

To date, seven KMT2 family proteins have been identified in human genes (KMT2A, KMT2B, KMT2C, KMT2D, KMT2E, KMT2E, SETD1A and SETD1B). Those of KMT2 proteins reside in large, multi-subunit complexes composed of unique sets of interacting proteins[13-16]. KMT2s have been reported to be associated with the carcinogenesis of gastric cancer. Koh $\mathrm{J}$ et al. reported that KMT2A served as a hypermutated status in the II and III GC of tumor microenvironment immune type, which suggests its effect on immune checkpoint therapy[17]. Howard L. McLeod et al. found that KMT2A mutation occurred in GC, and significant differences in protein-altering somatic mutation frequency in diverse geographic populations[18]. According to one study of next-generation sequencing (NGS) for advanced GC, the results show that KMT2B and KMT2D functioned as oncogenic drivers with the somatic mutation frequency was up to $11.5 \%$ and $15.4 \%$, respectively[19]. Meanwhile, KMT2D was examined to upregulated in GC tissues and depletion of KMT2D suppressed cell proliferation and induced apoptosis, which indicated KMT2D as an oncogene in GC[20]. In addition, highly somatic mutation frequency of KMT2C gene is reported to be occurred in GC but its effects on metastasis was different in some researches[21]. Sam S. Yoon et al. reported that KMT2C was frequently mutated in certain populations with diffuse-type gastric adenocarcinomas (DGA). KMT2C loss in DGA promoted epithelial-to-mesenchymal transition (EMT) and was associated with worse overall survival. Jaffer A Ajani et al. found that KMT2C mutation was the common cancer driver in primary gastric adenocarcinoma (DCA), and promoted peritoneal invasion of GCA especial for DGA via using whole-exome sequencing (WES) and whole transcriptome sequencing (RNA-seq) on 44 peritoneal carcinomatosis (PC) specimens (43 patients with PC) [22]. KMT2E is widely reported to be interaction with the development and progression in acute myeloid leukemia (AML) but its role in GC remains unknown[23]. Recent studies have revealed that KMT2F is involved in breast cancer and liver cancer development. Kwang Won Jeong et al. found that KMT2F was upregulated in breast cancer, and depletion of KMT2F in MCF-7 cells significantly decreased cell proliferation and migration, repressing tamoxifen-resistant via regulating miR-1915-13p[24]. Dongdong Lu et al. reported that long noncoding RNA CUDR cooperated with KMT2F to promote liver cancer growth and hepatocyte-like stem cell malignant transformation epigenetically[25]. Combined with the above findings, we recognize that KMT2s may be a significant oncogene or suppressor gene in various cancers. However, the underlying mechanism by which KMT2s are activated or inhibited, as well as the distinct functions of the KMT2s in GC have yet to be fully elucidated. Most of those genes are found with highly somatic mutation frequencies in GC based on the results of NGS but expression of KMT2s in tissues, the correlations with clinicopathological features and prognosis and the functional networks have not been reported in human GC. 
Here, we examined the correlation between the different KMT2s and GC based on bioinformatics analyses to explore their clinical significance and the functional networks in human GC. Expression of KMT2s in GC tissues and normal tissues, Correlation between KMT2s expression with clinicopathological characters and prognosis were examined by some databases including the Human Protein Atlas, UALCA, GEPIA and KMPLOT. Next, we analyzed the correlation between of KMT2s methylation with its expression and clinical data in GC. Finally, we further explore he mutations of different KMT2s in patients with GC to determine the functions, predicting the downstream kinase, miRNA and transcription factor targets.

\section{Results}

\section{Over-expression of different KMT2s family members in patients with GC}

To examine the potential correlation between the different KMT2s members and GC, we compared the TCGA data by using GEPIA database (http://gepia.cancer-pku.cn/) to explore the expression of KMT2s in cancer tissues and the normal tissues. We found that transcriptional levels of KMT2A/B/C/D had a trend to be higher in cancer tissues than the normal tissues, while transcriptional levels of KMT2E/F were significantly higher in cancer tissues (Figure 1A-B).

Having examined the mRNA expression patterns of KMT2s in GC, we tried to explore the protein expression patterns of KMT2s in GC by the Human Protein Atlas. As showed in Figure 2A-F, the expressions of KMT2A/B/C/D/E/F proteins were also higher in cancer tissues than the normal tissues. In summary, our results showed that transcriptional and proteomic expressions of KMT2s were overexpressed in patients with GC.

\section{Correlation between mRNA expressions of different KMT2s family members with the clinicopathological parameters of patients with GC}

Relationship between mRNA expressions of different KMT2s with clinicopathological features of GC patients were analyzed by using UALCAN (http://ualcan.path.uab.edu) including patients' individual cancer stages and tumor grades. Our results showed that $\mathrm{KMT} 2 \mathrm{~A} / \mathrm{B} / \mathrm{C} / \mathrm{D} / \mathrm{E} / \mathrm{F}$ mRNA expressions were significantly related with patients' individual cancer stages, and patients with advanced stages or grades tended to express higher mRNA of KMT2s. As showed in Figure 3, the highest mRNA expression of different KMT2s were found in stage 3. The reason why mRNA expressions of KMT2A/B/C/D/E/F in stage 3 seemed to be higher than that in stage 4 may be due to the small sample size. Consistent with the analysis of patients' individual cancer stages, our work found that mRNA expression of different KMT2s family members were also correlated with tumor grades. As tumor grades increased, the expression levels of KMT2s mRNA tended to be higher. As showed in Figure 4 A-F, the highest expression of KMT2A/B/D/F were all found in grade 3, while the highest expression of $\mathrm{KMT} 2 \mathrm{C} / \mathrm{E}$ were in grade 2 . Taken together, the results above indicated that mRNA expressions of $6 \mathrm{KMT} 2 \mathrm{~s}$ family members were significantly related with clinicopathological characteristics in GC patients.

\section{Correlation between methylation of KMT2s with its expression and clinical data in GC}

DNA methylation is a common heritable epigenetic modification in the genome of eukaryotic cells, plays an important role in regulating cell proliferation, differentiation, and individual development, and abnormal DNA methylation levels are closely related to tumor development. We used MEXPRESS to analyze the correlation between methylation of KMT2s with its expression and clinical data in GC. As showed in Figure $5 A, K M T 2 A$ expression was positively related with KMT2A promoter methylation $\left(r=0.448^{\star * *}\right)$, tumor stage $(p=0.01)$ and histological type $(p=4.311 e-5)$. In Figure 5B, KMT2B promoter methylation was positively related with KMT2B expression ( $r=0.479 * * *)$, tumor stage $(p=0.002)$ and histological type $(\mathrm{p}=0.002)$. In Figure $5 \mathrm{C}, \mathrm{KMT} 2 \mathrm{C}$ promoter methylation was positively related with $\mathrm{KMT} 2 \mathrm{C}$ expression ( $\mathrm{r}=0.429 \star \star \star)$, tumor stage $(p=0.018)$. In Figure 5D, KMT2D promoter methylation was positively related with KMT2D expression ( $\left.r=0.303^{\star * *}\right)$. In Figure 5E, KMT2CE promoter methylation was positively related with KMT2E expression ( $\left.r=0.403^{\star \star \star}\right)$, tumor stage $(p=0.014)$. In Figure 5F, KMT2F (SETD1A) promoter methylation was positively related with KMT2E expression $(r=0.357 * \star \star)$, tumor stage $(p=0.021)$ and histological type $(p=0.047)$. These findings indicated that high promoter methylation levels of KMT2A/B/C/D/E/F contributed normal expression of oncogenes (KMT2A/B/C/D/E/F) to induce the development and progression of GC.

\section{Prognostic values of expression levels of KMT2s mRNA in patients with GC}

We further explore the critical efficiency of KMT2s family members in the survival of patients with GC. Kaplan-Meier plotter (http://kmplot.com/analysis/) was performed to analyze the association between KMT2s mRNA expressions with the survival including overall survive (OS), progression-free survival (FP) and post-progression survival (PPS). The Kaplan-Meier curve and log rank test analyses revealed that patients of GC with higher of KMT2A/B/C/D/E/F mRNA expression levels have a shorter OS, FP and PPS in 150 months' follow up (Figure 6), $\mathrm{P}<0.05$. Thus, our results demonstrated that increased KMT2A/B/C/D/E/F expression were predicted to be associated with poor prognosis in GC. 
We used the cBioPortal online tools (https://www.cbioportal.org/) to analyze the KMT2s alterations, correlations and networks based on sequencing data from GC patients in the TCGA database. KMT2s were altered in 117 of 293 (40\%) GC patients. These alterations of $\mathrm{KMT} 2 \mathrm{~A} / \mathrm{B} / \mathrm{C} / \mathrm{D} / \mathrm{E} / \mathrm{F}$ were in the following (Table 1): KMT2A: amplification (2\%), mutation (10.6\%); KMT2B: amplification (2.4\%), homozygous (1.7\%) and mutation (7.5\%); KMT2C: amplification (0.7\%), homozygous (1.7\%) and mutation (15.4\%); KMT2D: amplification $(0.3 \%)$, homozygous $(1 \%)$ and mutation (21.8\%); KMT2E: amplification (2.7\%), homozygous $(0.7 \%)$ and mutation (5.8\%); KMT2F: amplification ( $0.3 \%$ ) and mutation (5.5\%). We also calculated the co-expression of KMT2s with each other for stomach adenocarcinoma, and Pearson's correction was included. We found significant and positive correlations in the followings KMT2s『KMT2A with KMT2C, KMT2D and $\mathrm{KMT} 2 \mathrm{E}$; KMT2B with KMT2D and KMT2F; KMT2C with KMT2A and KMT2E; KMT2D with KMT2A, KMT2B and KMT2F; KMT2E with KMT2A and KMT2C; KMT2F with KMT2B and KMT2D (Figure 7C). Next, we constructed the network for KMT2s and the neighboring genes with the alteration frequency $>5 \%$ (Figure 7D).

\section{Predicted functions and pathways of the changes in KMT2s and their frequently altered neighboring genes in patients with GC}

The functions of KMT2s and the genes significant correlated with KMT2s alterations were predicted by analyzing GO and KEGG pathways in the database for STRING (https://string-db.org/). To address this, we used the KMT2s-neighboring genes that were altered at frequencies > $5 \%$ in the GC TCGA database (Figure 7D and Table 1). As showed in Figure 8, analysis of significantly enriched GO terms suggested that those proteins were mainly located in (G0:0005654) nucleoplasm, (G0:0005694) chromosome, (G0:0035097) histone methyltransferase complex, (G0:0043231) intracellular membrane-bounded organelle, (G0:0044451) nucleoplasm part and (G0:0043232) intracellular nonmembrane-bounded organelle. These proteins are primarily involved in (G0:0034968) histone lysine methylation, (GO:0006325) chromatin organization, (GO:0018193) peptidyl-amino acid modification, (G0:0006355) regulation of transcription, DNA-templated and (G0:0051252) regulation of RNA metabolic process. while they also serve as (GO:0018024) histone-lysine N-methyltransferase activity, (G0:0042800) histone methyltransferase activity (H3-K4 specific), (G0:0140100) transferase activity, (GO:0140110) transcription regulator activity and (G0:0003677) DNA binding. Moreover, KEGG pathway analysis showed enrichment in (hsa00310) lysine degradation, (hsa05226) gastric cancer such as MAPK and PI3K/AKT pathways, (hsa05210) colorectal cancer, (hsa04550) signaling pathways regulating pluripotency of stem cells and (hsa04934) Cushing's syndrome. Among these significant pathways, pathways of lysine degradation and gastric cancer were regulated by KMT2s in GC (Figure 9A-B).

\section{KMT2s networks of kinase, miRNA and transcription factor targets in GC}

To further predict the targets of KMT2s in GC, we analyzed the kinase, miRNA and transcription factor targets generated by Gene Set Enrichment Analysis (GSEA). The top 5 most significant target networks of KMT2A/B/C/D/E/F were included in Table 2, respectively. Importantly, we found that all of the $6 \mathrm{KMT} 2 \mathrm{~s}$ had common kinas targets: AKT and MAPK1, which suggested KMT2s might be involved in pathways such as MAPK and PI3K/AKT signaling pathway.

\section{Discussion}

Cancer genetic and epigenetic alterations are reported to play an important role in the development and progression of gastric cancer (GC) [26]. KMT2s family of genes mutations are involved in many cancers. Although the role of KMT2s as oncogenic drivers of several cancers have been partially confirmed, its clinical significance and functional networks in GC are known with limitation in literature. In this study, we used bioinformatics analysis to elaborate the effects of KMT2A/B/C/D/E/F on the tumorigenesis and prognosis of GC.

Previous studies have identified KMT2A and KMT2B (KMT2A/B) as oncogenes in several cancers including breast cancer, colorectal cancer, pancreatic cancer, and acute myeloid leukemia[27]. Thus, KMT2A/B are of great significance in the cancer research. Somatic mutation frequency of KMT2A/B was found in GC though sequencing method, but its clinical value and biological function of KMT2A/B in GC has not been reported. Our result that KMT2A/B were upregulated in GC, and their overexpression were negatively related with tumor stages and grades. Patients with lower KMT2A/B benefited with good prognosis in OS, FP and PPS. The above results first revealed the clinical significance of KMT2A/B in GC, expanding the knowledge of KMT2A/B applied for clinical transformation. Also, our work analyzed GO and KEGG pathway of KMT2A/B in GC, and predicted the kinase, miRNA and transcription factor targets in the development of GC. These findings indicated the potential mechanism of KMT2A/B in regulating the development and progression of GC.

Among KMT2s, KMT2C and KMT2D (KMT2C/D) are the 2 most studied in gastric cancer. Silencing KMT2C in non-transformed HFE-145 gastric epithelial cells promoted EMT, while KMT2C re-expression in the DGA cell lines MKN-45 and SNU-668 could inhibited the EMT[21]. However, another paper published on "GUT" performed whole-exome sequencing (WES) and whole transcriptome sequencing (RNA-seq) on $44 \mathrm{PC}$ specimens (43 patients with PC), and found that alterations associated with aggressive PC phenotypes emerged with increased

Page $4 / 19$ 
mutation in KMT2C[22]. In our study, we also identified KMT2C as hypermutation in $\mathrm{GC}$, and patients with increased KMT2C expression suffered poor OS, FP and FFP. These results were consistent with the paper published in "GUT", but more experiments in details needed to be performed. KMT2D was found to be highly expressed in GC tissues, and related with poor survival. Depletion of KMT2D suppressed cell proliferation and induced apoptosis. Similarity to the above results, we further confirmed the clinical value of KMT2D in GC though bioinformatics analysis, suggesting KMT2D as a novel oncogene for prognosis and optimal treatment of GC patients.

Up to now, little was known about the expression and role of KMT2E and KMT2F (KMT2E/F) in GC. In our report, the expression levels of $\mathrm{KMT} 2 \mathrm{E}$ and KMT2F in gastric cancer tissues were significantly higher than that in normal tissues. Advanced tumor stages and grades tended to express higher KMT2E/F mRNA levels. Interestingly, high KMT2E and KMT2F expression were significantly correlated with poor OS, FP and PPS in all of the patients with gastric cancer, indicating the role of these KMT2s as oncogenic drivers in GC. No studies have yet reported the function networks of KMT2E and KMT2F in the development of GC. Though bioinformatics analysis, we revealed the GO and KEGG pathway of those KMT2s family proteins and also predicted the potential kinase, miRNA and transcription factor targets in GC. These findings provide the knowledge about the mechanism of KMT2s in GC. However, further studies needed to be carried via cellular or animal experiments to verify the ideas.

DNA methylation is an important epigenetic modification, mainly occurring in the $\mathrm{CpG}$ island of DNA and its changes at the single gene level and genome level play an important role in tumor development. Increased DNA methylation levels of oncogenes or decreased DNA methylation levels of tumor suppressor genes may lead to tumorigenesis[28]. In this study, we found that the promoter methylation levels of $\mathrm{KMT} 2 \mathrm{~A} / \mathrm{B} / \mathrm{C} / \mathrm{D} / \mathrm{E} / \mathrm{F}$ were significantly associated with $\mathrm{KMT} 2 \mathrm{~A} / \mathrm{B} / \mathrm{C} / \mathrm{D} / \mathrm{E} / \mathrm{F}$ expression in $\mathrm{GC}$, and also found to be related with tumor stage. The above results revealed that the hypermethylation levels of oncogenes predicted the high risk for patients with higher KMT2A/B/C/D/E/F expression in the development of GC, and improve the therapeutic strategies for patients as well.

Significantly, KMT2A/B/C/D/E/F were predicted to have two common meaningful kinases targets: AKT and MAPK, which indicated that KMT2s were involved in MAPK and PI3K/AKT signaling pathways. Consistent with the above results, we also found that KMT2A/B/C/D/E/F were markedly associated with gastric cancer pathway such as MAPK and PI3K/AKT pathways though GEAS analysis. Thus, $\mathrm{KMT} 2 \mathrm{~A} / \mathrm{B} / \mathrm{C} / \mathrm{D} / \mathrm{E} / \mathrm{F}$ might regulate MAPK and PI3K/AKT pathways by interacting with common targets of AKT and MAPK to inhibit GC occurrence.

In conclusion, results of our study showed that increased expression of mRNA and protein were found in all of the 6 KMT2s family of genes in GC tissues, and KMT2s mRNA expression were related with patients' individual cancer stages and tumor grades in patients with GC. Meanwhile, the promoter methylation levels of KMT2s were found to be significantly positively related with KMT2s expression in GC. In addition, patients with higher KMT2A/B/C/D/E/F expression were associated with shorter OS, FP and PPS. And high somatic mutation frequency $(40 \%)$ of KMT2s were observed in GC. Furthermore, we used the significant neighboring genes of KMT2s to explore the GO and KEGG pathway, the results showed that biological process such as histone lysine methylation and chromatin organization, cellular component such as nucleoplasm and chromosome, molecular function such as histone-lysine N-methyltransferase activity and DNA binding, KEGG pathway such as lysine degradation and gastric cancer such as PI3K/AKT and MAPK pathways were markedly regulated by KMT2s mutation in GC. Finally, we predicted the network of kinase AKT and MAPK targets commonly regulated by KMT2s in GC,which also suggested that KMT2s may regulate PI3K/AKT and MAPK pathways to influence the development and progression of GC. Thus, our study demonstrated that data mining efficiently revealed information about the clinical value of KMT2s and potential regulatory networks in GC, laying a foundation for further study of the role KMT2s in carcinogenesis.

\section{Materials And Methods GEPIA Database}

GEPIA (http://gepia.cancer-pku.cn/) is an online tool for analyzing the RNA sequencing expression data of 9,736 tumors and 8,587 normal samples from the Cancer Genome Atlas and the Genotype Tissue Expression (GTEx) projects[29]. GEPIA provides customizable functions such as tumor/normal differential expression analysis, profiling according to cancer types or pathological stages, patient survival analysis, similar gene detection, correlation analysis and dimensionality reduction analysis.

\section{The Human Protein Atlas}

The Human Protein Atlas (https://www.proteinatlas.org) is a publicly available database, which provides tissue and cell distribution information containing all 24,000 human proteins[30]. We analyzed the protein expression of KMT2s in GC tissues and the normal tissues via immunohistochemistry (IHC) analysis.

\section{UALCAN Analysis}


UALCAN is publicly available at http://ualcan.path.uab.edu. It provides easy access to publicly available cancer OMICS data (TCGA and MET500) from 31 cancer types, which contains graphs and plots depicting gene expression and patient survival information based on gene expression[31]. We used this database to analyze the association between KMT2s mRNA expression with individual cancer stages and tumor stages.

\section{The Kaplan-Meier Plotter}

The prognostic value of mRNA expression of distinct KMT2s in gastric cancer was analyzed by using Kaplan- Meier plotter (http://kmplot.com/analysis/), in which information about association of gene expression with survival of patients of liver cancer, breast cancer, ovarian cancer, lung cancer and gastric cancer could be easily access to. To analyze the OS, FP, and PPS of patients with gastric cancer, patients were divided into high and low expression group based on median values of mRNA expression and validated by K-M survival curves, with the hazard ratio (HR) with $95 \%$ confidence intervals (Cls) and log rank p_value. Statically significant difference was considered when a $p$ value $<0.05$.

\section{MEXPRESS Analysis}

MEXPRESS (https://mexpress.be/) is a data visualization tool designed for the easy visualization of TCGA expression, DNA methylation and clinical data, as well as the relationships between them. We used this database to analyze the effect of methylation of KMT2s expression on the development of GC.

\section{cBioPortal Analysis}

The cBioPortal (http://cbioportal.org), currently containing 225 cancer studies, is an open-access resource for interactive exploration of multidimensional cancer genomics datasets[32]. We used cBioPortal to analyze KMT2s alterations in the TCGA GC dataset, which containing 293 samples. The search parameters included OncoPrint, Cancer Type Summary, Mutations, Co-expression, Network and so on. The tab OncoPrint shows an overview of KMT2s alterations per sample in total $293 \mathrm{GC}$ samples. Network tab reflects the biological functional network of KMT2s interacting with neighboring genes acquired from public pathway databases, with filter options and colorcoding based on the frequency of genomic alterations in each gene. Next, we chose KMT2s and the neighboring genes with alteration frequency $>5 \%$ to reveal the GO and KEGG pathways in CRC via STRING analysis.

\section{GO and KEGG analysis}

Functions of KMT2s mutations and the significant neighboring genes with mutation rates $>5 \%$ were chosen to analyze by GO and KEGG in the Database for STRING (https://string-db.org/). GO enrichment analysis can predict the functional roles of KMT2s mutations and chosen neighboring genes on the basis of three aspects, including biological processes(BP), cellular components (CC), and molecular functions (MF), while KEGG analysis can define the pathways regulated by KMT2s in GC.

\section{Analysis for The Networks of Kinase, miRNA and Transcription Factor Targets}

LinkedOmics is publicly available portal (http://www.linkedomics.org/login.php) that includes multi-omics data from all 32 TCGA cancer types. We used the LinkInterpreter module predicted its interacted target networks of kinases, miRNA, and transcription factors though gene set enrichment analysis (GSEA) analysis. The rank criterion was a $\mathrm{P}<0.05$, and 500 simulations were performed.

\section{Declarations}

\section{AUTHOR CONTRIBUTIONS}

L-Q.Y performed the experiments. L-S.Q, C-Y.O and X-Y analyzed the data. S-X.H wrote the manuscript. W-Y.B is the guarantor of this work, had full access to all data reported in the study, and takes responsibility for the integrity of the data and the accuracy of the data analysis.

\section{CONFLICTS OF INTERESTS}

The authors declare no conflicts of interests.

\section{FUNDING}

This work is supported by Grants from the National Natural Science Foundation of China (NO. No.81472620)

\section{References}


1. Bray F, Ferlay J, Soerjomataram I, Siegel RL, Torre LA, Jemal A. Global cancer statistics 2018: globocan estimates of incidence and mortality worldwide for 36 cancers in 185 countries. CA: A Cancer Journal for Clinicians. 2018; 68: 394-424.

2. Siegel RL, Miller KD, Jemal A. Cancer statistics. 2019. CA: A Cancer Journal for Clinicians. 2019; 69: 7-34.

3. Thrift AP, El-Serag HB. Burden of gastric cancer. Clin Gastroenterol Hepatol. 2019.

4. Shilatifard A. Chromatin modifications by methylation and ubiquitination: implications in the regulation of gene expression. Annu Rev Biochem. 2006;75:243-69.

5. Schuettengruber B, Martinez A, lovino N, Cavalli G. Trithorax group proteins: switching genes on and keeping them active. Nat Rev Mol Cell Bio. 2011;12:799-814.

6. Rao RC, Dou Y. Hijacked in cancer: the kmt2 (mll) family of methyltransferases. Nat Rev Cancer. 2015;15:334-46.

7. Li J, Ivansson E, Klevebring D, Tobin NP, Lindström LS, Holm J, Prochazka G, Cristando C, Palmgren J, Törnberg S, Humphreys K, Hartman J, Frisell J, et al. Molecular differences between screen-detected and interval breast cancers are largely explained by pam50 subtypes. Clin Cancer Res. 2017;23:2584-92.

8. Tan TZ, Rouanne M, Tan KT, Huang RY, Thiery J. Molecular subtypes of urothelial bladder cancer: results from a meta-cohort analysis of 2411 tumors. Eur Urol. 2019;75:423-32.

9. Chang S, Yim S, Park H. The cancer driver genes idh1/2, jarid1c/ kdm5c, and utx/ kdm6a: crosstalk between histone demethylation and hypoxic reprogramming in cancer metabolism. Experimental \& Molecular Medicine. 2019; 51.

10. Sharma A, Jyotsana N, Gabdoulline R, Heckl D, Kuchenbauer F, Slany RK, Ganser A, Heuser M. Mn1 is indispensable for mll-rearranged acute myeloid leukemia. Haematologica. 2019: 2018-211201.

11. Puccini A, Lenz HJ, Marshall JL, Arguello D, Raghavan D, Korn WM, Weinberg BA, Poorman K, Heeke AL, Philip PA, Shields AF, Goldberg RM, Salem ME. Impact of patient age on molecular alterations of left-sided colorectal tumors. Oncologist. 2019;24:319-26.

12. Chen Y, Xu J, Skanderup AJ, Dong Y, Brannon AR, Wang L, Won HH, Wang PI, Nanjangud GJ, Jungbluth AA, Li W, Ojeda V, Hakimi AA, et al. Molecular analysis of aggressive renal cell carcinoma with unclassified histology reveals distinct subsets. Nat Commun. $2016 ; 7$.

13. Nakamura T, Mori T, Tada S, Krajewski W, Rozovskaia T, Wassell R, Dubois G, Mazo A, Croce CM, Canaani E. All-1 is a histone methyltransferase that assembles a supercomplex of proteins involved in transcriptional regulation. Mol Cell. 2002;10:1119-28.

14. Cho Y, Hong T, Hong S, Guo H, Yu H, Kim D, Guszczynski T, Dressler GR, Copeland TD, Kalkum M, Ge K. Ptip associates with mll3- and mll4-containing histone h3 lysine 4 methyltransferase complex. J Biol Chem. 2007;282:20395-406.

15. Lee J, Skalnik DG. Cpg-binding protein (cxxc finger protein 1) is a component of the mammalian set1 histone h3-lys4 methyltransferase complex, the analogue of the yeast set1/compass complex. J Biol Chem. 2005;280:41725-31.

16. Lee J, Tate CM, You J, Skalnik DG. Identification and characterization of the human set1b histone h3-lys4 methyltransferase complex. J Biol Chem. 2007;282:13419-28.

17. Koh J, Nam SK, Roh H, Kim J, Lee BC, Kim JW, Ahn SH, Park DJ, Kim HH, Park KU, Chung JH, Kim WH, Lee HS. Somatic mutational profiles of stage ii and iii gastric cancer according to tumor microenvironment immune type. Genes Chromosom Cancer. 2018;58:12-22.

18. Jia F, Teer JK, Knepper TC, Lee JK, Zhou H, He Y, McLeod HL. Discordance of somatic mutations between asian and caucasian patient populations with gastric cancer. Mol Diagn Ther. 2017;21:179-85.

19. Ge S, Li B, Li Y, Li Z, Liu Z, Chen Z, Wu J, Gao J, Shen L. Genomic alterations in advanced gastric cancer endoscopic biopsy samples using targeted next-generation sequencing. Am J Cancer Res. 2017;7:1540-53.

20. Xiong W, Deng Z, Tang Y, Deng Z, Li M. Downregulation of kmt2d suppresses proliferation and induces apoptosis of gastric cancer. Biochem Bioph Res Co. 2018;504:129-36.

21. Cho S, Yoon C, Lee JH, Chang KK, Lin J, Kim Y, Kook M, Aksoy BA, Park DJ, Ashktorab H, Smoot DT, Schultz N, Yoon SS. Kmt2C mutations in diffuse-type gastric adenocarcinoma promote epithelial-to-mesenchymal transition. Clin Cancer Res. 2018;24:6556-69.

22. Wang R, Song S, Harada K, Ghazanfari Amlashi F, Badgwell B, Pizzi MP, Xu Y, Zhao W, Dong X, Jin J, Wang Y, Scott A, Ma L, et al. Multiplex profiling of peritoneal metastases from gastric adenocarcinoma identified novel targets and molecular subtypes that predict treatment response. Gut. 2019: 2018-318070.

23. Lucena-Araujo AR, Coelho-Silva JL, Pereira-Martins DA, Silveira DR, Koury LCDA, Melo RAM, Bittencourt R, Pagnano K, Pasquini R, Nunes EC, Fagundes EM, Gloria AB, Kerbauy FR, et al. Combining gene mutation with gene expression analysis improves outcomes prediction in acute promyelocytic leukemia. Blood. 2019: 2019000239.

24. Jin ML, Kim YW, Jin HL, Kang H, Lee EK, Stallcup MR, Jeong KW. Aberrant expression of setd1a promotes survival and migration of estrogen receptor a-positive breast cancer cells. Int J Cancer. 2018;143:2871-83. 
25. Li T, Zheng Q, An J, Wu M, Li H, Gui X, Pu H, Lu D. Set1a cooperates with cudr to promote liver cancer growth and hepatocyte-like stem cell malignant transformation epigenetically. Mol Ther. 2016;24:261-75.

26. Slavin TP, Weitzel JN, Neuhausen SL, Schrader KA, Oliveira C, Karam R. Genetics of gastric cancer: what do we know about the genetic risks? Translational Gastroenterology Hepatology. 2019;4:55.

27. Mousavian Z, Nowzari-Dalini A, Rahmatallah Y, Masoudi-Nejad A. Differential network analysis and protein-protein interaction study reveals active protein modules in glucocorticoid resistance for infant acute lymphoblastic leukemia. Mol Med. $2019 ; 25$.

28. Fattahi S, Golpour M, Amjadi-Moheb F, Sharifi-Pasandi M, Khodadadi P, Pilehchian-Langroudi M, Ashrafi GH, Akhavan-Niaki H. Dna methyltransferases and gastric cancer: insight into targeted therapy. Epigenomics-Uk. 2018;10:1477-97.

29. Tang Z, Li C, Kang B, Gao G, Li C, Zhang Z. Gepia: a web server for cancer and normal gene expression profiling and interactive analyses. Nucleic Acids Res. 2017;45:W98-102.

30. Thul PJ, Lindskog C. The human protein atlas: a spatial map of the human proteome. Protein Sci. 2018;27:233-44.

31. Chandrashekar DS, Bashel B, Balasubramanya SAH, Creighton CJ, Ponce-Rodriguez I, Chakravarthi BVS, K, Varambally S. Ualcan: a portal for facilitating tumor subgroup gene expression and survival analyses. Neoplasia. 2017;19:649-58.

32. Gao J, Aksoy BA, Dogrusoz U, Dresdner G, Gross B, Sumer SO, Sun Y, Jacobsen A, Sinha R, Larsson E, Cerami E, Sander C, Schultz N. Integrative analysis of complex cancer genomics and clinical profiles using the cbioportal. Sci Signal. 2013;6:I1.

\section{Tables}

Table 1. The Mutations of The KMT2s and their significant Neighboring Genes with Alteration Rates $>5 \%$ in Gastric Cancer (cBioPortal) 


\begin{tabular}{|lllll|}
\hline Genes & Total Alteration & Amplification & Homozygous & Mutation \\
\hline APC & $17.4 \%$ & $0.3 \%$ & $3.1 \%$ & $14.3 \%$ \\
\hline ASH1L & $11.9 \%$ & $2.4 \%$ & 0 & $9.6 \%$ \\
\hline ATR & $6.8 \%$ & $1.4 \%$ & 0 & $5.5 \%$ \\
\hline BCL9 & $7.2 \%$ & $0.7 \%$ & 0 & $6.5 \%$ \\
\hline BCL9L & $8.5 \%$ & $1 \%$ & 0 & $7.8 \%$ \\
\hline CTNNB1 & $8.2 \%$ & $1.4 \%$ & $0.7 \%$ & $6.1 \%$ \\
\hline EHMT1 & $6.5 \%$ & $2 \%$ & 0 & $4.4 \%$ \\
\hline EMHT2 & $7.2 \%$ & $1.4 \%$ & $1.7 \%$ & $4.1 \%$ \\
\hline KMT2A & $12.3 \%$ & $2 \%$ & 0 & $10.6 \%$ \\
\hline KMT2B & $11.6 \%$ & $2.4 \%$ & $1.7 \%$ & $7.5 \%$ \\
\hline KMT2C & $17.4 \%$ & $0.7 \%$ & $1.7 \%$ & $15.4 \%$ \\
\hline KMT2D & $23.2 \%$ & $0.3 \%$ & $1 \%$ & $21.8 \%$ \\
\hline KMT2E & $9.2 \%$ & $2.7 \%$ & $0.7 \%$ & $5.8 \%$ \\
\hline KMT5B & $7.8 \%$ & $3.8 \%$ & $0.7 \%$ & $3.4 \%$ \\
\hline NCOA2 & $9.2 \%$ & $3.4 \%$ & $0.3 \%$ & $5.8 \%$ \\
\hline NSD1 & $8.2 \%$ & $1 \%$ & $1 \%$ & $6.1 \%$ \\
\hline NSD2 & $6.8 \%$ & $0.3 \%$ & $1.4 \%$ & $5.1 \%$ \\
\hline NSD3 & $7.5 \%$ & $1 \%$ & $1 \%$ & $5.5 \%$ \\
\hline SETD1A & $5.8 \%$ & $0.3 \%$ & 0 & $5.5 \%$ \\
\hline SETD2 & $7.2 \%$ & 0 & $1.7 \%$ & $5.5 \%$ \\
\hline SETDB1 & $9.2 \%$ & $4.1 \%$ & 0 & $5.1 \%$ \\
\hline SETDB2 & $5.1 \%$ & $0.7 \%$ & $1 \%$ & $3.4 \%$ \\
\hline TCF & $5.5 \%$ & $0.3 \%$ & $2 \%$ & $3.1 \%$ \\
\hline TCFL2 & $5.5 \%$ & $1 \%$ & $0.7 \%$ & $3.8 \%$ \\
\hline
\end{tabular}

Table 2. Networks of The Top 5 Kinase, miRNA and Transcription Factor Targets of KMT2A/B/C/D/E/F in Gastric Cancer 


\begin{tabular}{|c|c|c|c|c|c|c|c|}
\hline Genes & Kinase Target & miRNA Target & TF Target & Genes & Kinase Target & miRNA Target & TF Target \\
\hline \multirow[t]{5}{*}{ KMT2A } & Kinase_MAPK1 & $\begin{array}{l}\text { TGAATGT,MIR- } \\
181\end{array}$ & V\$GATA6_01 & \multirow[t]{5}{*}{ KMT2D } & Kinase_GRK3 & $\begin{array}{l}\text { CTCAGGG,MIR- } \\
125\end{array}$ & V\$E2F1_Q6_01 \\
\hline & Kinase_AKT1 & $\begin{array}{l}\text { TGCACTT,MIR- } \\
519\end{array}$ & V\$SFI1_01 & & Kinase_CDK2 & $\begin{array}{l}\text { CTGAGCC,MIR- } \\
24\end{array}$ & VSE2F_Q4 \\
\hline & Kinase_GSK3B & $\begin{array}{l}\text { TTGCACT,MIR- } \\
130\end{array}$ & V\$SRY_02 & & Kinase_AKT1 & $\begin{array}{l}\text { TTGCCAA,MIR- } \\
182\end{array}$ & VSE2F_Q3_01 \\
\hline & Kinase_PRKACB & $\begin{array}{l}\text { ATGTACA,MIR- } \\
493\end{array}$ & V\$SRF_Q6 & & Kinase_ATM & $\begin{array}{l}\text { CCCAGAG,MIR- } \\
326\end{array}$ & V\$SE2F_Q6 \\
\hline & Kinase_PRKX & $\begin{array}{l}\text { CAGTGTT,MIR- } \\
141\end{array}$ & V\$SOX9_B1 & & Kinase_MAPK1 & $\begin{array}{l}\text { TGCTTTG,MIR- } \\
330\end{array}$ & V\$SE2F1_Q4_01 \\
\hline \multirow[t]{5}{*}{ KMT2B } & Kinase_CDK2 & $\begin{array}{l}\text { GTGCCTT,MIR- } \\
506\end{array}$ & V\$SLK1_02 & \multirow[t]{5}{*}{ KMT2E } & Kinase_GRK3 & $\begin{array}{l}\text { GCACTTT,MIR- } \\
\text { 17-5P }\end{array}$ & V\$NRF1_Q6 \\
\hline & Kinase_AKT1 & $\begin{array}{l}\text { ACCAAAG,MIR- } \\
9\end{array}$ & V\$NRF1_Q6 & & Kinase_AKT1 & $\begin{array}{l}\text { TTTGCAC,MIR- } \\
19\end{array}$ & V\$HFH4_01 \\
\hline & Kinase_MAPK1 & $\begin{array}{l}\text { CTACCTC,MIR- } \\
98\end{array}$ & V\$AP2_Q6 & & Kinase_CDK2 & $\begin{array}{l}\text { CAGTATT,MIR- } \\
429\end{array}$ & V\$OCT1_03 \\
\hline & Kinase_CHEK1 & $\begin{array}{l}\text { CACTGCC,MIR- } \\
449\end{array}$ & V\$OLF1_01 & & Kinase_MAPK1 & $\begin{array}{l}\text { TGAATGT,MIR- } \\
181\end{array}$ & V\$SRY_02 \\
\hline & Kinase_ATM & $\begin{array}{l}\text { CTCAGGG,MIR- } \\
125\end{array}$ & V\$AP2 & & Kinase_CSNK2A1 & $\begin{array}{l}\text { TGCACTT,MIR- } \\
519\end{array}$ & V\$FOX04_01 \\
\hline \multirow[t]{5}{*}{ KMT2C } & Kinase_GRK3 & $\begin{array}{l}\text { AAGCACA,MIR- } \\
218\end{array}$ & V\$̦ELK1_02 & \multirow[t]{5}{*}{$\mathrm{KMT} 2 \mathrm{~F}$} & Kinase_CDK2 & $\begin{array}{l}\text { CTCAGGG,MIR- } \\
125\end{array}$ & V\$AP2GAMMA_01 \\
\hline & Kinase_CSNK2A1 & $\begin{array}{l}\text { ACACTGG,MIR- } \\
199\end{array}$ & V\$MMF2_02 & & Kinase_AKT1 & $\begin{array}{l}\text { TGAATGT,MIR- } \\
181\end{array}$ & V\$AP2ALPHA_01 \\
\hline & Kinase_MAPK3 & $\begin{array}{l}\text { AGCACTT,MIR- } \\
93\end{array}$ & V\$NRF1_Q6 & & Kinase_MAPK1 & $\begin{array}{l}\text { CTGAGCC,MIR- } \\
24\end{array}$ & V\$E2F1_Q6_01 \\
\hline & Kinase_MAPK1 & $\begin{array}{l}\text { AGGCACT,MIR- } \\
\text { 515-3P }\end{array}$ & V\$MYC_Q2 & & Kinase_CHEK1 & $\begin{array}{l}\text { TTTTGAG,MIR- } \\
373\end{array}$ & V\$SP1_Q2_01 \\
\hline & Kinase_AKT1 & $\begin{array}{l}\text { ATACTGT,MIR- } \\
144\end{array}$ & V\$GABP_B & & Kinase_ATM & $\begin{array}{l}\text { TAGCTTT,MIR- } \\
9\end{array}$ & V\$SP1_Q6_01 \\
\hline
\end{tabular}

Figures 
A

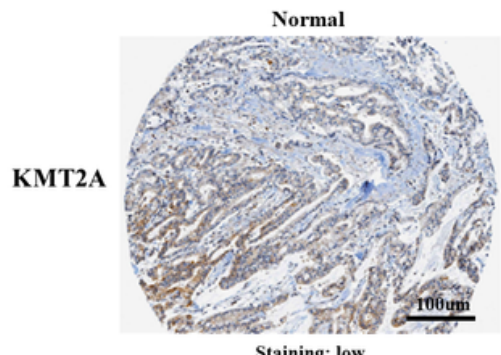

Staining: low
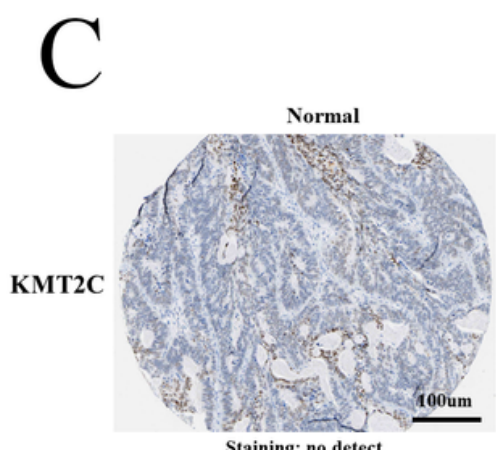

$\mathrm{E}$

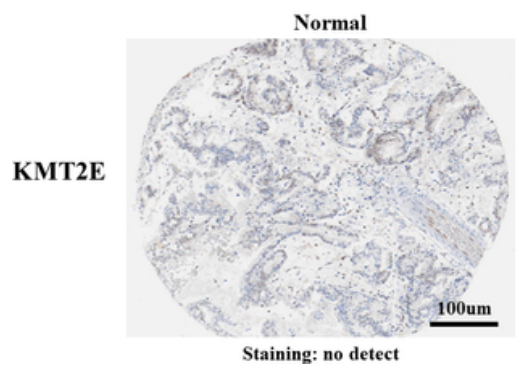

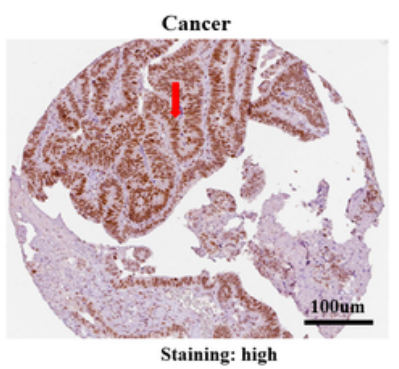

Staining: higl

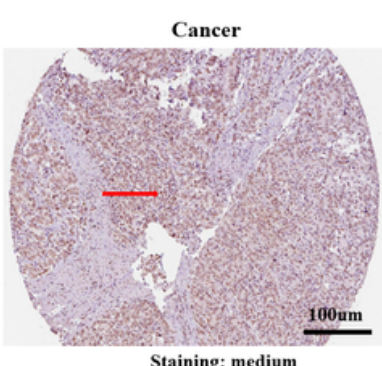

Staining: medium

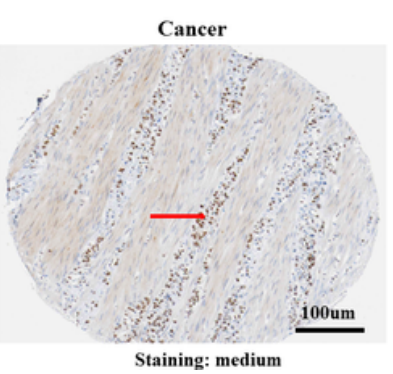

B
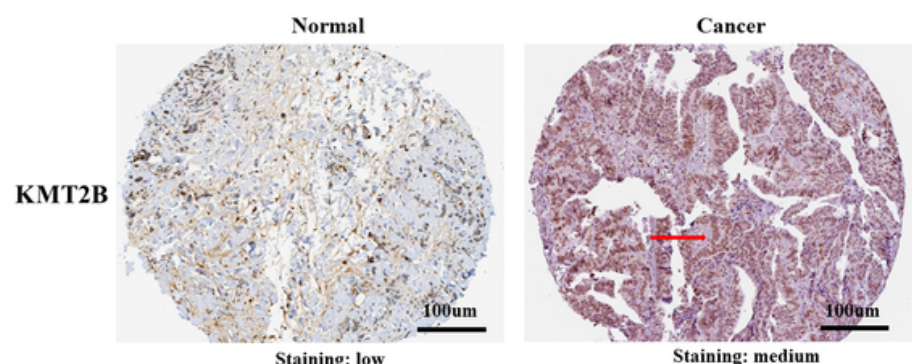

$\mathrm{D}$

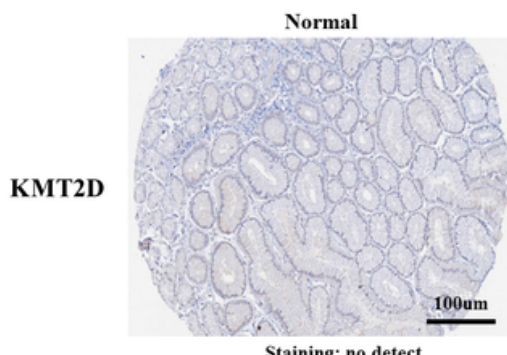

Staining: no detect

F
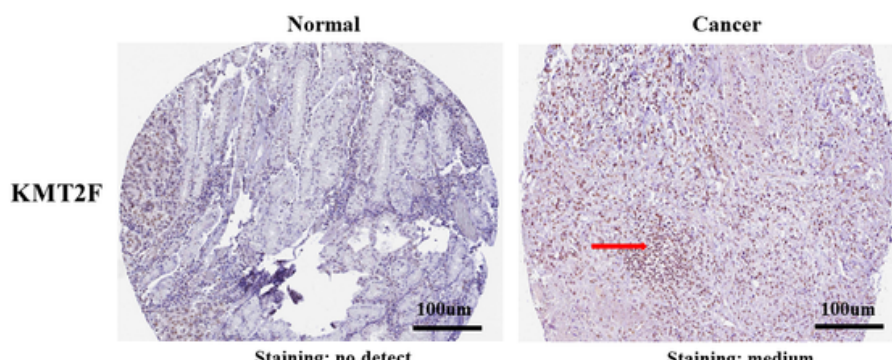

Figure 1

The Expression Levels of KMT2s Proteins in Gastric Cancer (The Human Protein Atlas). (A-F) Compared with the normal tissues, $\mathrm{KMT} 2 \mathrm{~A} / \mathrm{B} / \mathrm{C} / \mathrm{D} / \mathrm{E} / \mathrm{F}$ were highly expressed in cancer tissues in $\mathrm{IHC}$ analysis. 
A

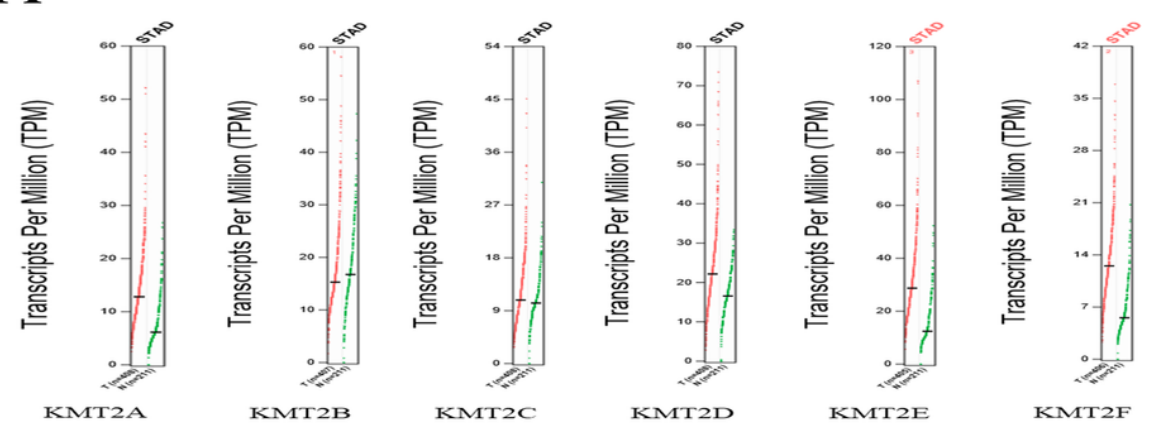

$\mathrm{B}$
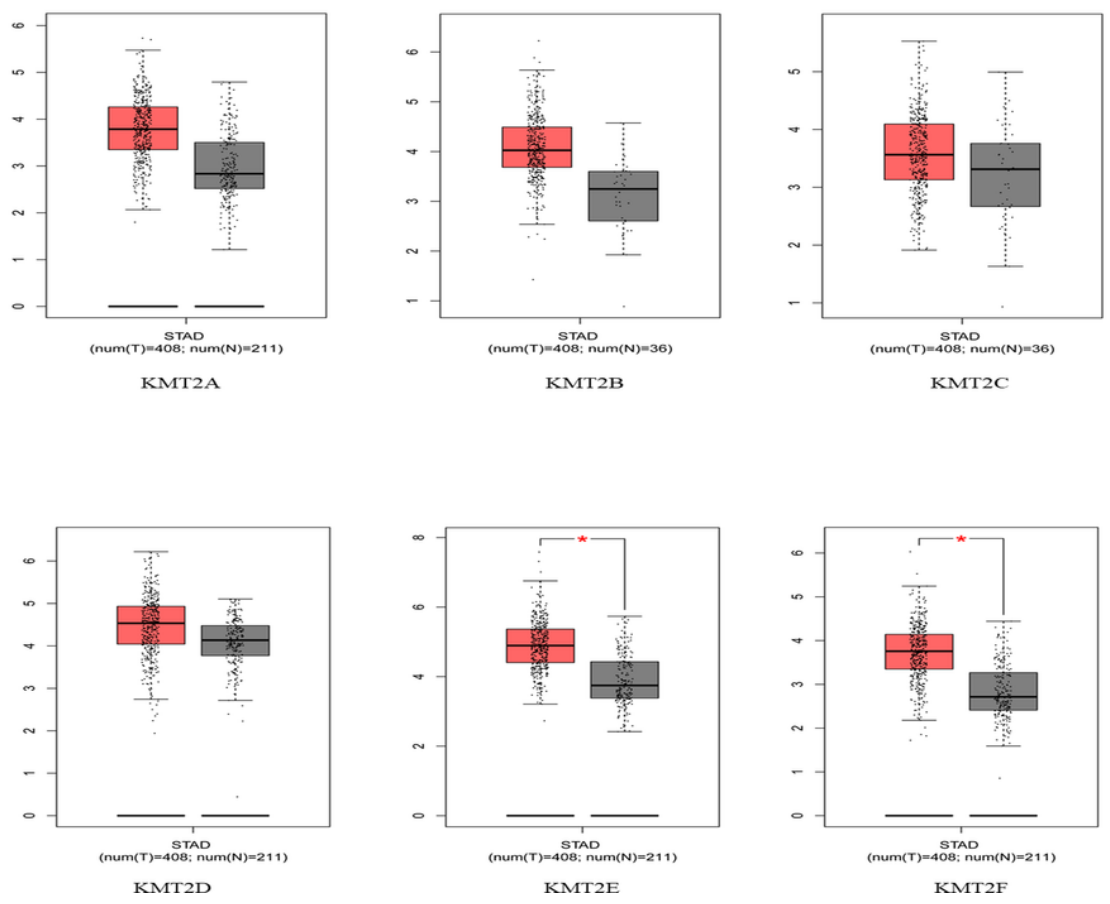

Figure 2

The Expression of KMT2s in Gastric Cancer (GEPIA). (A-B) mRNA expression of 6 KMT2s family member were found to be over-expressed in $\mathrm{GC}$ tissues compared to the normal tissues in profile and boxplot. 
A

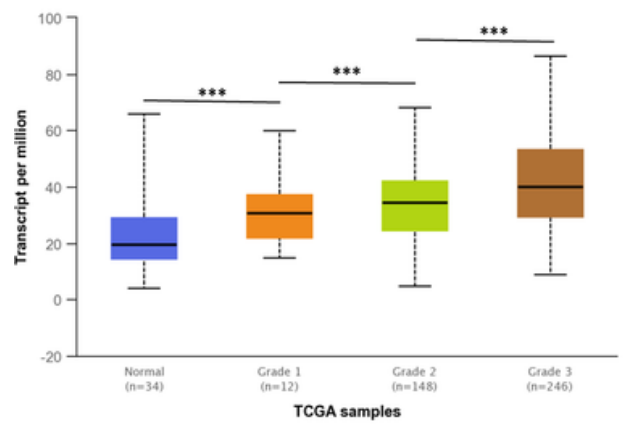

KMT2A
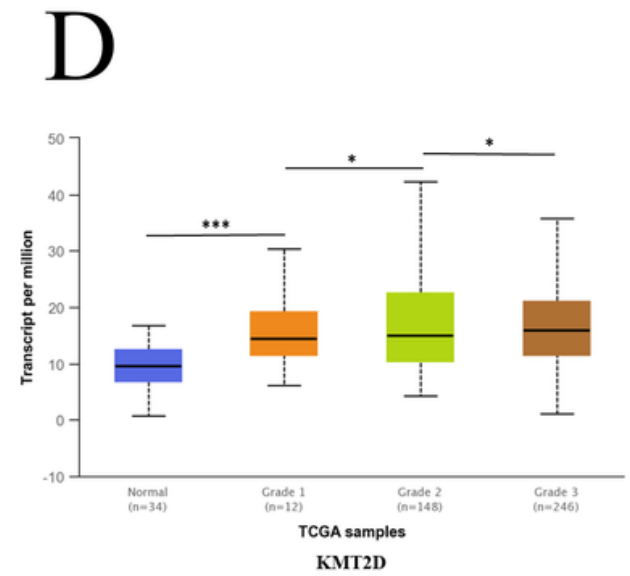

$\mathrm{B}$
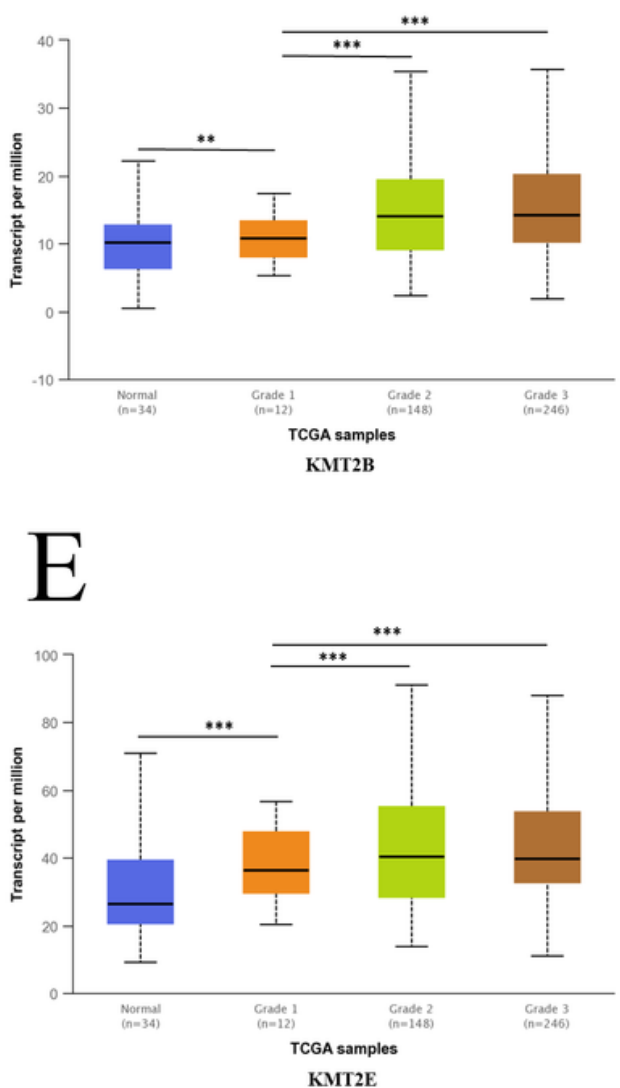

C

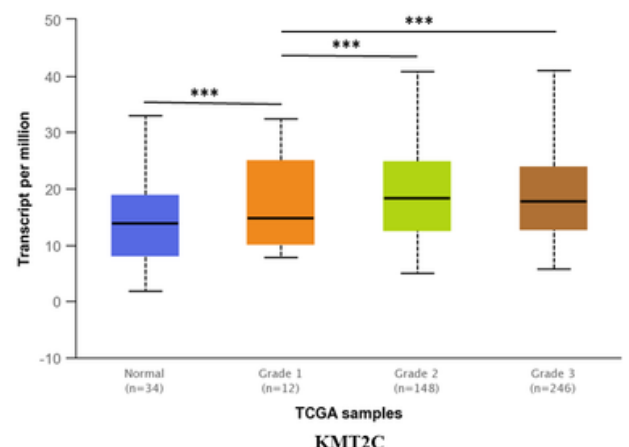

$\mathrm{F}$

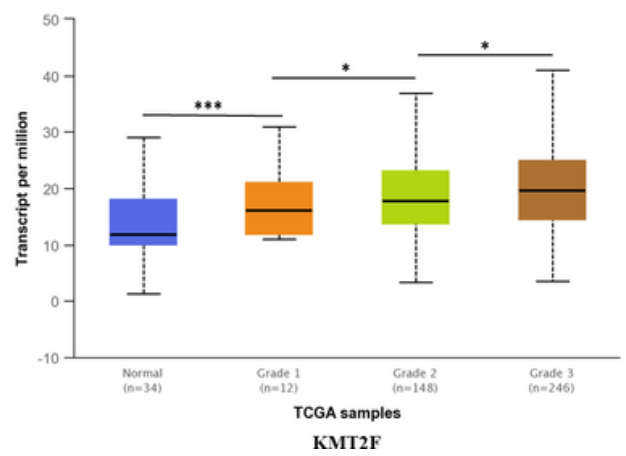

Figure 3

Correlation between mRNA Expression of Distinct KMT2s Family Members and Tumor Grades of GC Patients (UALCAN). mRNA expression of $\mathrm{KMT} 2 \mathrm{~A} / \mathrm{B} / \mathrm{C} / \mathrm{D} / \mathrm{E} / \mathrm{F}$ were found to be associated with patients' tumor grades, patients who were in advanced grades tended to express higher expression of KMT2s. ${ }^{*} p<0.05,{ }^{*} \mathrm{p}<0.01,{ }^{* \star *} \mathrm{p}<0.001$. 

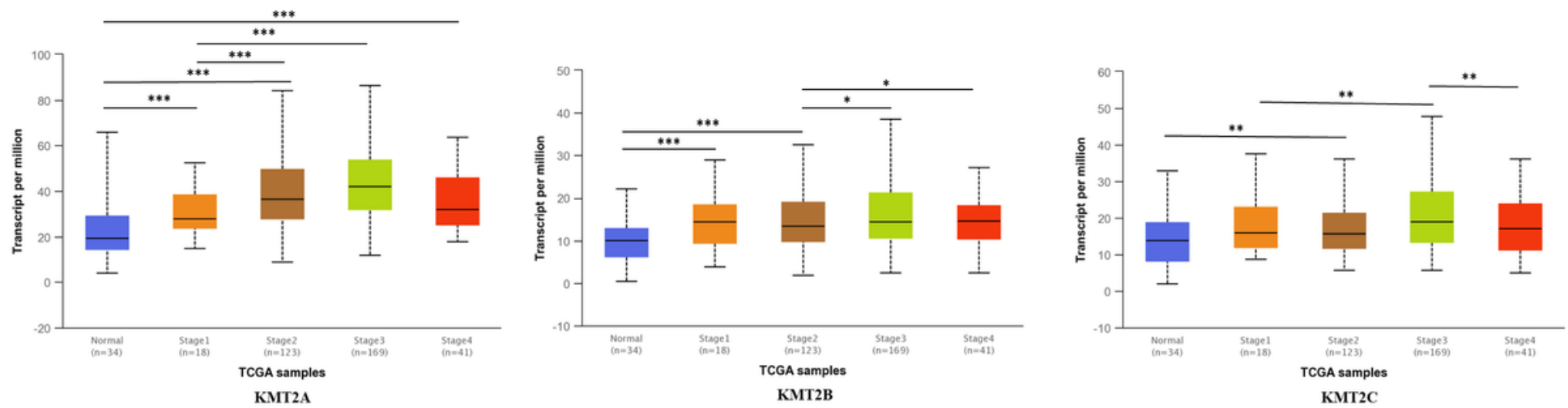

$\mathrm{D}$

$\mathrm{E}$

$\mathrm{F}$
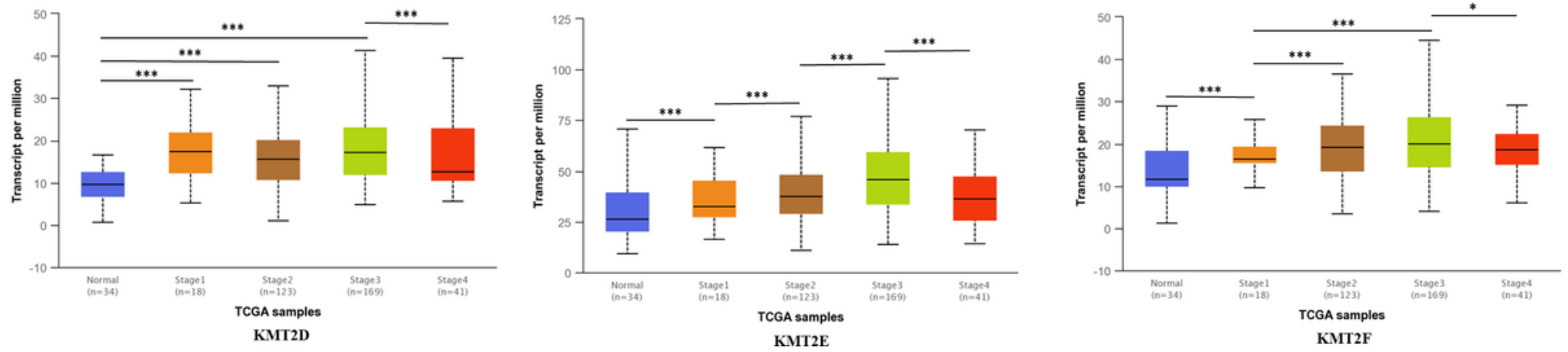

Figure 4

Relationship between mRNA Expression of Distinct KMT2s Family Members and Individual Cancer Stages of GC Patients (UALCAN). (A-F) mRNA expression of KMT2 A/B/C/D/E/F were found to be associated with patients' individual cancer stages, patients who were in advanced stages tended to express higher expression of KMT2s. ${ }^{*} p<0.05,{ }^{*} \mathrm{p}<0.01,{ }^{* \star *} \mathrm{p}<0.001$. 


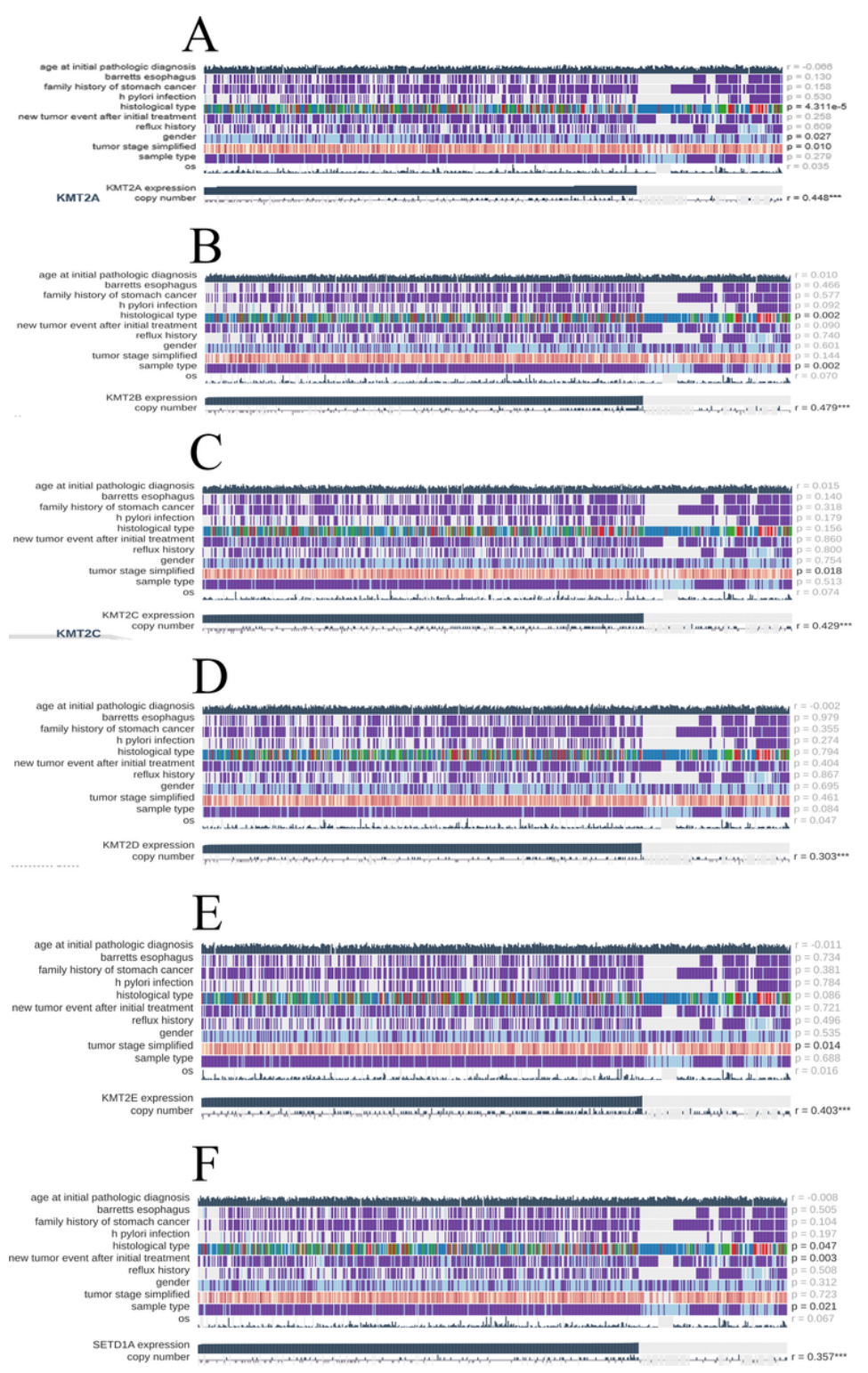

\section{Figure 5}

Correlation between the Promoter Methylation Levels of KMT2s with Its Expression and Clinical Data in GC (MEXPRESS) (A) KMT2A expression was positively related with KMT2A promoter methylation ( $\left.r=0.448^{\star \star \star}\right)$, tumor stage $(p=0.01)$ and histological type $(p=4.311 e-5)$. (B) KMT2B promoter methylation was positively related with KMT2B expression $(r=0.479 * \star *)$, tumor stage $(p=0.002)$ and histological type $(p=0.002)$. (C) KMT2C promoter methylation was positively related with KMT2C expression ( $r=0.429 * * *)$, tumor stage ( $p=0.018)$. (D) KMT2D promoter methylation was positively related with KMT2D expression $\left(r=0.303^{\star * \star}\right)$. (E) KMT2CE promoter methylation was positively related with KMT2E expression ( $\left.r=0.403^{* * *}\right)$, tumor stage $(\mathrm{p}=0.014)$. ( $\left.\mathrm{F}\right) \mathrm{KMT} 2 \mathrm{~F}(\mathrm{SETD} 1 \mathrm{~A})$ promoter methylation was positively related with $\mathrm{KMT} 2 \mathrm{E}$ expression $\left(r=0.357^{\star \star \star}\right)$, tumor stage $(p=0.021)$ and histological type $(p=0.047)$. 
A

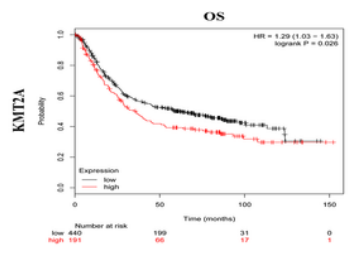

B

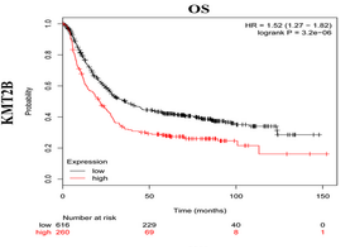

C

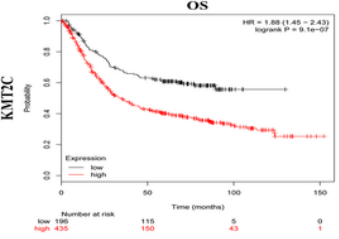

D

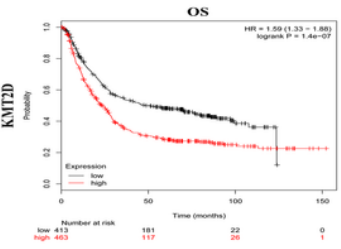

E
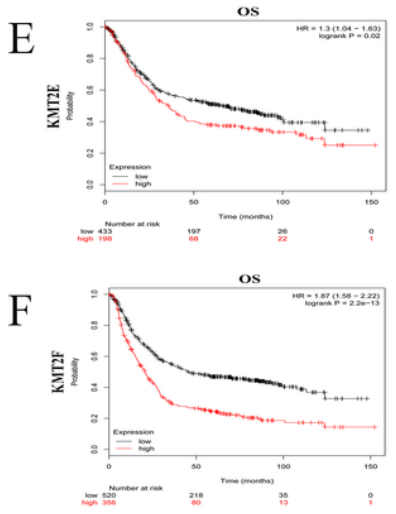
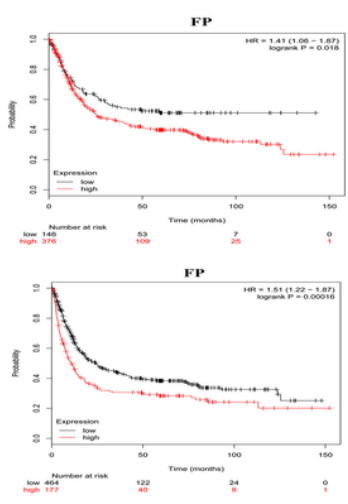

FP
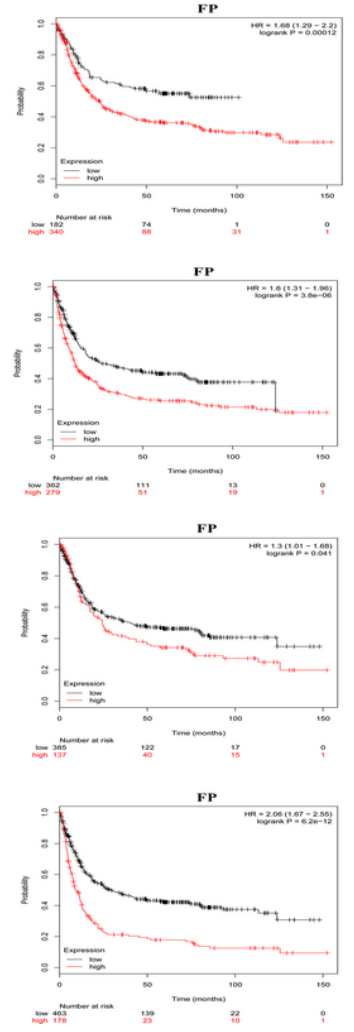

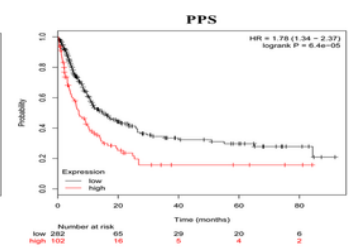

PPS

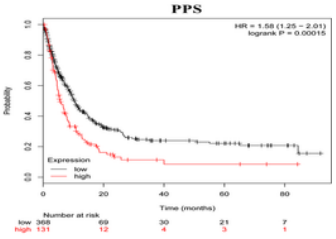

pips

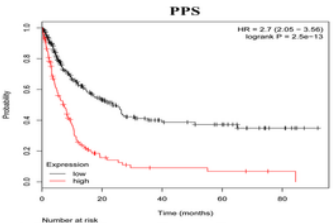

PPS
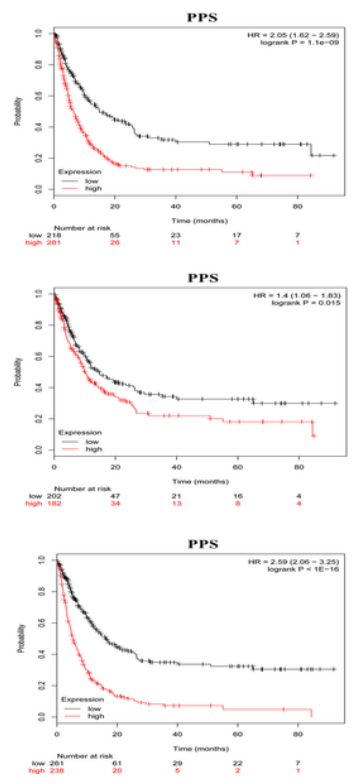

Figure 6

The prognostic value of KMT2A/B/C/D/E/F in Gastric Cancer (Kaplan-Meier Plotter). (A-F) Survival analysis revealed that patients with higher KMT2A/B/C/D/E/F mRNA expression had shorter OS, FP and PPF. 
Altered in $117(40 \%)$ of 293 sequenced cases/patients (293 total)
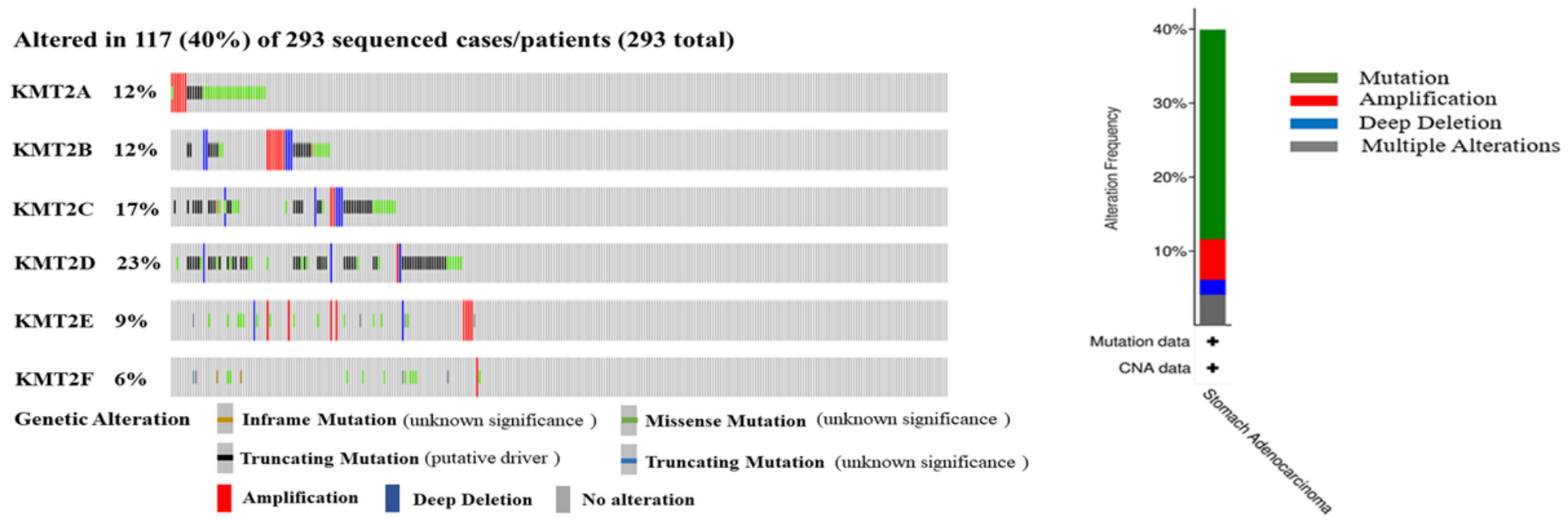

$\mathrm{C}$

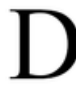

\begin{tabular}{|c|c|c|c|c|c|c|c|}
\hline 1 & 0.21 & 0.632 & 0.322 & 0.609 & 0.195 & KMT2A & Network view of the KMT2s neighboring genes in GC \\
\hline 0.21 & 1 & 0.0787 & 0.438 & 0.0105 & 0.566 & KMT2B & \\
\hline 0.632 & 0.0787 & 1 & 0.239 & 0.692 & 0.133 & KMT2C & \\
\hline 0.322 & 0.438 & 0.293 & 1 & 0.111 & 0.598 & KMT2D & \\
\hline 0.609 & 0.0105 & 0.692 & 0.111 & 1 & 0.0193 & KMT2E & \\
\hline 0.195 & 0.566 & 0.133 & 0.598 & 0.0193 & 1 & KMT2F & \\
\hline KMT2A & KMT2B & KMT2C & KMT2D & KMT2E & KMT2F & & \\
\hline
\end{tabular}

Figure 7

KMT2s Gene Expression and Mutation Analysis in Gastric Cancer (cBioPortal). (A and B) KMT2s gene expression and mutation analysis in gastric cancer. (C) Correlation between KMT2s with each other in gastric cancer. (D) The network of KMT2s and its neighboring genes with mutation rates $>5 \%$. 
A

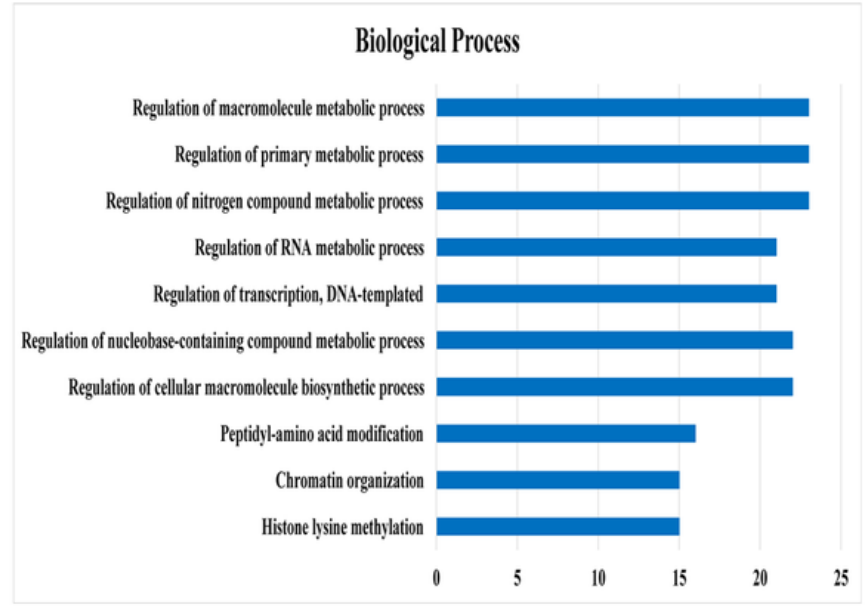

$\mathrm{C}$

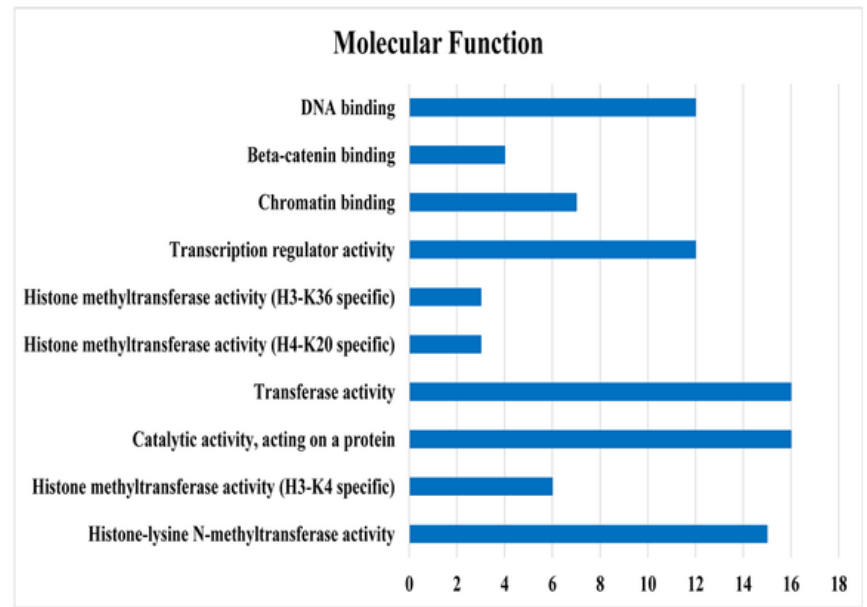

$\mathrm{B}$

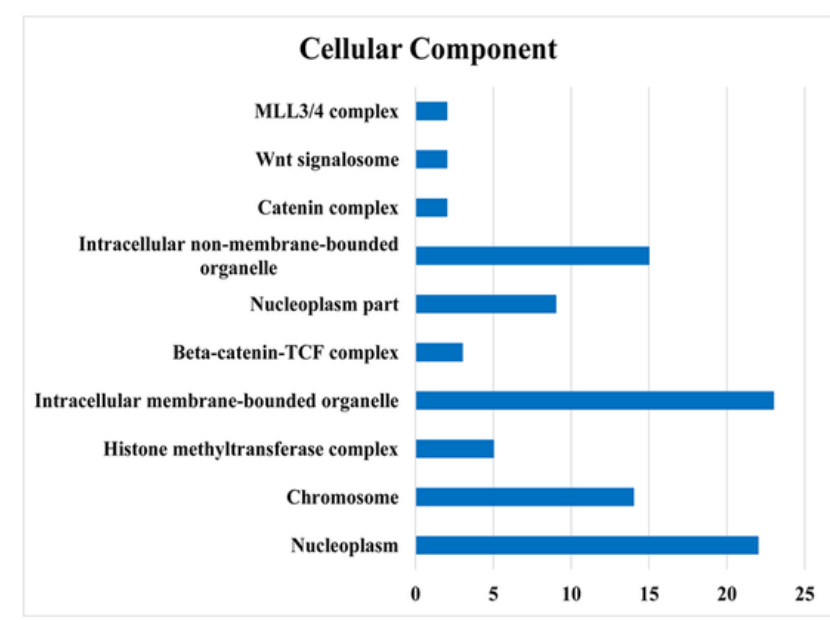

$\mathrm{D}$

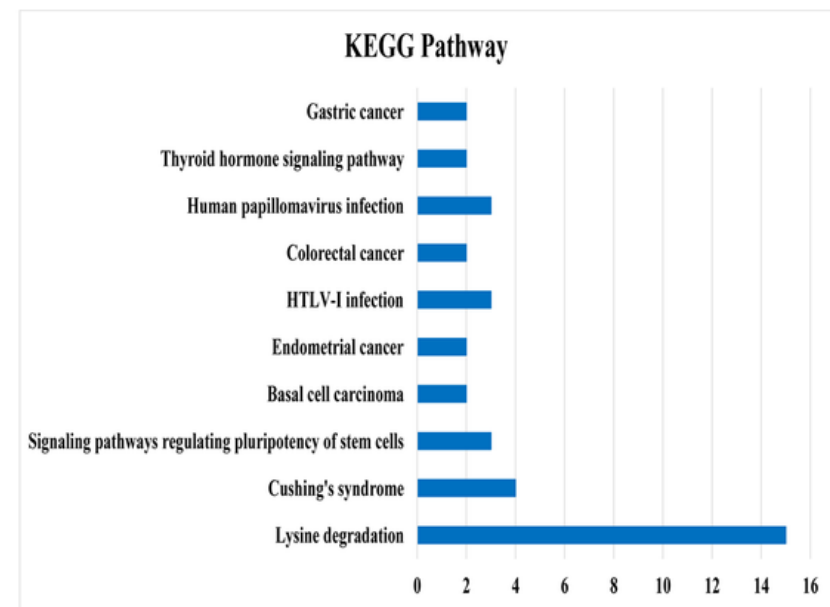

\section{Figure 8}

Predicted the Functions of the Mutations in KMT2s and Their Significant Neighboring Genes with Alteration Rates $>5 \%$ in Gastric Cancer. (A-

C) GO functional enrichment analysis predicted three main functions of KMT2s mutations and their significant altered neighbor genes with alteration rates $>5 \%$, including biological process, cellular components and molecular functions. (D) KEGG pathways of KMT2s in GC. 
A

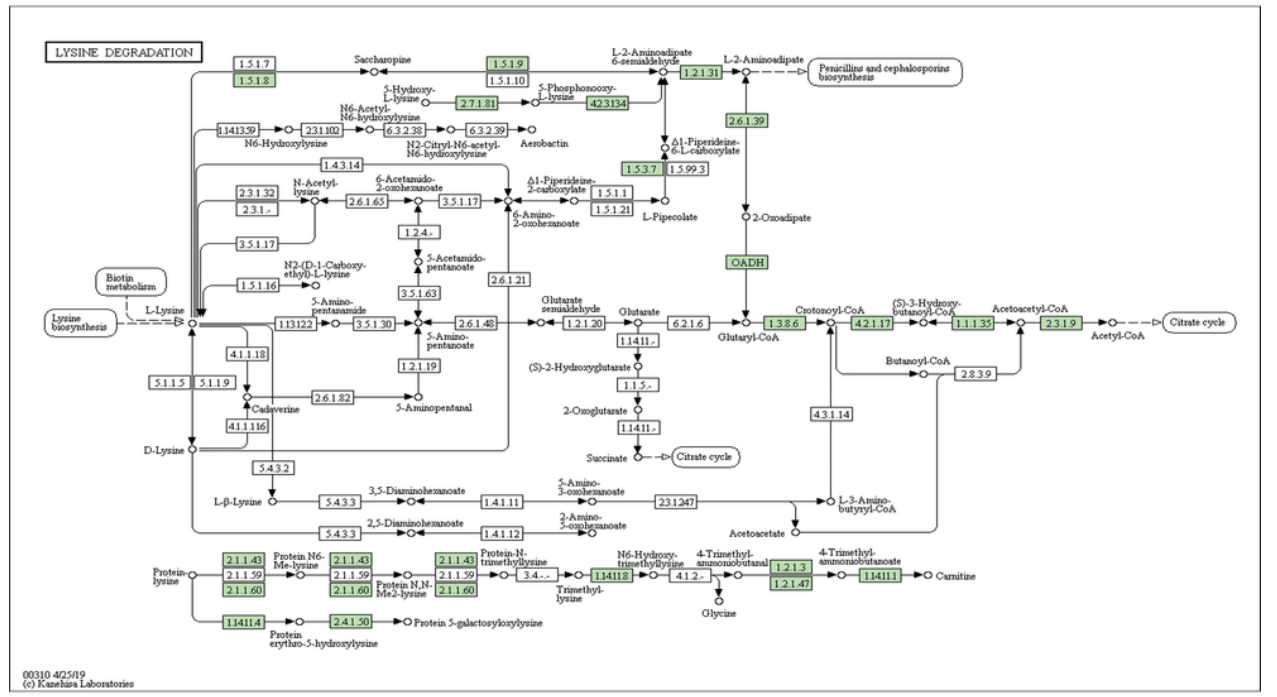

$\mathrm{B}$

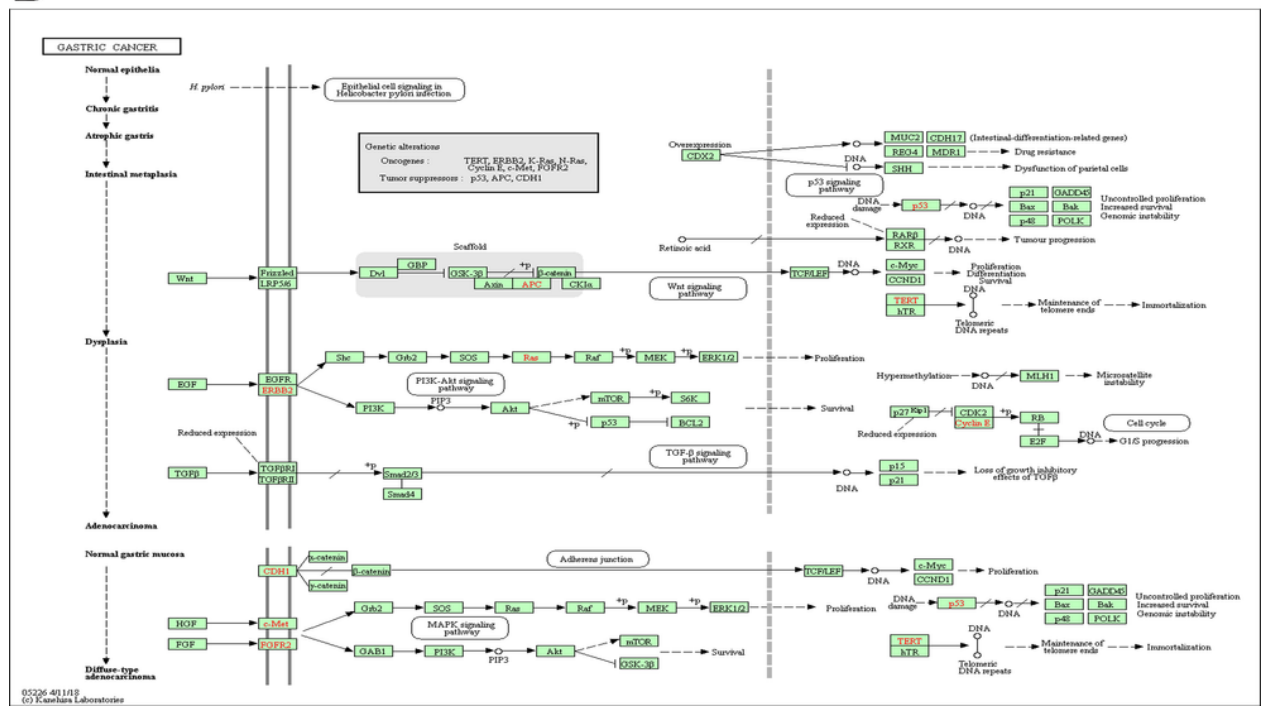

\section{Figure 9}

Lysine Degradation and Gastric Cancer Pathways Regulated by the KMT2s Alteration in Gastric Cancer. (A) Lysine degradation pathway. (B) Gastric cancer pathway such as PI3K/AKT and MARK signaling pathways. 